\title{
Spectral imaging of multi-color chromogenic dyes in pathological specimens
}

\author{
Merryn V.E. Macville ${ }^{\mathrm{a}, \mathrm{b}, *}$, \\ Jeroen A.W.M. Van der Laak ${ }^{a}$, \\ Ernst J.M. Speel ${ }^{\mathrm{c}}$, Nir Katzir ${ }^{\mathrm{d}}$, Yuval Garini ${ }^{\mathrm{d}}$, \\ Dirk Soenksen $^{\mathrm{e}}$, George McNamara ${ }^{\mathrm{f}}$, \\ Peter C.M. de Wilde ${ }^{\mathrm{a}}$, \\ Antonius G.J.M. Hanselaar ${ }^{\mathrm{a}}$, \\ Anton H.N. Hopman ${ }^{\mathrm{c}}$ and Thomas Ried ${ }^{\mathrm{b}}$ \\ ${ }^{a}$ Department of Pathology, University Medical Center \\ St Radboud, Nijmegen, The Netherlands \\ ${ }^{\mathrm{b}}$ Department of Genetics, Division of Clinical \\ Sciences, National Cancer Institute/NIH, Bethesda, \\ $M D, U S A$ \\ ${ }^{\mathrm{c}}$ Department of Molecular Cell Biology, University of \\ Maastricht, Maastricht, The Netherlands \\ ${ }^{\mathrm{d}}$ Applied Spectral Imaging, Migdal Ha'Emek, Israel \\ eAperio Technologies, Carlsbad, CA, USA \\ ${ }^{\mathrm{f}}$ Children's Hospital Research Institute, Los Angeles, \\ CA, USA
}

Received 19 October 2000

Accepted 20 February 2001

We have investigated the use of spectral imaging for multicolor analysis of permanent cytochemical dyes and enzyme precipitates on cytopathological specimens. Spectral imaging is based on Fourier-transform spectroscopy and digital imaging. A pixel-by-pixel spectrum-based color classification is presented of single-, double-, and triple-color in situ hybridization for centromeric probes in T24 bladder cancer cells, and immunocytochemical staining of nuclear antigens Ki-67 and TP53 in paraffin-embedded cervical brush material (AgarCyto). The results demonstrate that spectral imaging unambiguously identifies three chromogenic dyes in a single bright-field microscopic specimen. Serial microscopic fields from the same specimen can be analyzed using a spectral reference library. We conclude that spectral imaging of multi-color chromogenic dyes is a reliable and robust method for pixel color recognition and classification. Our data fur-

\footnotetext{
${ }^{*}$ Corresponding author: Dr. Merryn V.E. Macville, Laboratory for ISH, Department of Pathology, University Medical Center St Radboud, P.O. Box 9101, 6500 HB Nijmegen, The Netherlands. E-mail: macville@mailbox.kun.nl.
}

ther indicate that the use of spectral imaging (a) may increase the number of parameters studied simultaneously in pathological diagnosis, (b) may provide quantitative data (such as positive labeling indices) more accurately, and (c) may solve segmentation problems currently faced in automated screening of cell- and tissue specimens. htm.

Figures on http://www.esacp.org/acp/2001/22-3/macville.

Keywords: Spectral imaging, Fourier-transform spectroscopy, multicolor, in situ hybridization, immunocytochemistry, bright-field microscopy, absorption, optical density, pathology

\section{Abbreviations}

SKY, spectral karyotyping; ICC, immunocytochemistry; ISH, in situ hybridization; RGB, red-, green, and blue; HE, hematoxylin and eosin; PO, peroxidase; AP, alkaline phosphatase; DAB, diaminobenzidine; AEC, amino-ethylcarbazole; TMB, tetramethylbenzidine; FR, Fast Red; NF, New Fuchsin; nm, nanometer, $10^{-9} \mathrm{~m}$; OD, optical density; SPY, spectral pathology; $\mathrm{CCD}$, charge coupled device.

\section{Introduction}

Spectral imaging of fluorescent dyes has been successfully applied to spectral karyotyping (SKY) for the genome-wide screening of chromosomal aberrations in metaphase cells [18], and is now being used in clinical and tumor cytogenetics [7,19,20,25]. Dedicated software for SKY and spectral FISH enables analysis of combinatorial labeled nucleic acid probes in metaphase and interphase cells, respectively. Spectral imaging may also be used for conventional transmission light microscopy using chromogenic dyes [10,16]. Such an application might be of great clinical importance, because diagnosis of neoplasms frequently requires application of immunocytochemistry (ICC) and in situ hybridization (ISH) for the assessment of phenotypic and genetic biomarkers to complement the cy- 
tomorphologic diagnosis [1,2,14]. Enzyme-mediated cytochemical detection procedures for bright-field microscopic analysis of cell- and tissue preparations have been under continuous development in the last decades. In the mid 1990s technical breakthroughs have been made for multi-color bright-field analysis of ISH, ICC, and combinations thereof [4,13,21,22]. Bright-field microscopy optics can be used to detect up to three ISH targets simultaneously, together with a nuclear or cytoplasmic dye for morphology assessment. Examination of a higher number of ISH or ICC markers is hampered by broad absorption spectra of enzyme precipitates which makes color discrimination by eye or color cameras difficult. Advances in digital image analysis based on three-chip CCD red-, green-, and blue- (RGB) color camera images enable discrimination of up to three different stains simultaneously $[9,15,24,26]$. However, color recognition of weak or small ISH signals, such as for chromosome locusspecific probes, has been impossible.

The aim of this study was to explore spectral imaging for the analysis of bright-field multi-color interphase ISH and ICC in combination with cytomorphological staining in specimens of T24 human transitional cell carcinoma cells, spermatozoids, and cervical scrapings.

\section{Materials and methods}

\subsection{Sample preparation}

Multi-color ISH experiments were performed on T24 human transitional cell carcinoma cell line obtained from ATCC (Manassas, Virg.), and spermatozoids obtained from a healthy male (provided by the Department of Obstetrics \& Gynecology, University Maastricht, The Netherlands). ICC experiments were performed on an AgarCyto preparation [6] of a cervical scraping collected at the Department of Obstetrics \& Gynecology, University Nijmegen, The Netherlands.

T24 cells were cultured, trypsinized, and fixed in suspension with $70 \%$ ethanol. Cell preparations were made by applying a few drops of the cell suspension to a microscopic slide. Pretreatment steps for removal of cytoplasm and improving accessibility of ISH reagents were performed as described [5,23]. Single-, double and triple ISH experiments on T24 cells utilized centromeric alpha-satellite probes for chromosome 1, 7, and 15 . Spermatozoids were fixed in $70 \%$ ethanol and smeared on a microscopic slide. Pretreatment and ISH procedures with probes for $\mathrm{X}$ - and Y-chromosomespecific loci were essentially as described [13]. All probes were labeled by nick-translation with biotindUTP, digoxigenin-dUTP, or FITC-dUTP according to manufacturer's directions (Boehringer-Mannheim).

The novel AgarCyto cell-embedding technique [6] was applied to a cervical scraping from a woman with severe dysplasia. Cells obtained from the cervical brush were fixed with Unifix (Klinipath, Duiven, The Netherlands) and subsequently embedded in agarose and paraffin. Four- $\mu \mathrm{m}$ thick sections were cut for staining.

\subsection{In situ hybridization, immunocytochemistry, and cytomorphological staining}

Chromogenic dyes included the enzyme-substrates diaminobenzidine (DAB, brown; Sigma), aminoethylcarbazole (AEC, red-brown; Sigma), tetramethylbenzidine (TMB, green; Sigma), Fast Red (FR, red; Sigma) and New Fuchsin (NF, red; Sigma). Counterstains included hematoxylin, eosin, and eosin-derivative Diff-Quik (Baxter, Utrecht, The Netherlands).

We used spectral imaging on specimens subjected to (a) single-color ISH without counterstaining, (b) single-color ISH with hematoxylin counterstaining, (c) double-color ISH without counterstaining, (d) doublecolor ISH with Diff-Quik counterstaining, (e) triplecolor ISH without counterstaining, (f) hematoxylin and eosin (HE)-staining, and (g) single-color immunocytochemistry with hematoxylin counterstaining.

All ISH procedures were essentially performed as described in a technical review [4]. Immunocytochemistry of AgarCyto specimens was performed as described previously [6].

For single-color ISH of T24 cells, a biotin-labeled probe was detected with peroxidase (PO)-conjugated avidin (Vector, USA) and visualized with PO-substrate DAB, AEC or TMB. Alternately, probes were detected with alkaline phosphatase (AP)-conjugated avidin (Vector) and visualized with AP-substrate FR or NF. For double- or triple labeling of T24 cells, the first and second probes were labeled with biotin and digoxigenin, respectively. Double-color ISH experiments were visualized consecutively as described for single ISH in the combinations DAB/AEC, DAB/TMB and $\mathrm{DAB} / \mathrm{NF}$. The triple-color ISH experiment was visualized in DAB, TMB and NF. The third probe was labeled with FITC-dUTP and detected with mouse antiFITC monoclonal antibody (Dako, Denmark) followed by AP-conjugated anti-mouse (Boehringer, Germany). 
In double- and triple-color detection, antibodies were selected on epitope specificity and on host animal to prevent interspecies reactivity between the antibodies of the different detection systems. Furthermore, enzyme activity was blocked before proceeding to the next antibody detection and enzyme reaction [22]. Nuclei were counterstained with hematoxylin, except in preparations for pure dye spectra measurements and triple-color ISH.

For double-color ISH of spermatozoids, the chromosome $\mathrm{X}$ probe was labeled with biotin, and the chromosome $\mathrm{Y}$ probe with digoxigenin (Boehringer) and visualized with DAB and TMB, respectively, as described $[12,13]$. Sperm structures were counterstained with Diff-Quik.

Immunocytochemical staining of AgarCyto cell block specimens were performed with a mouse antiTP53 monoclonal antibody (DO-7; Novocastra, Newcastle, UK) and a mouse anti-Ki67 monoclonal antibody (MIB-1; Dianova, Hamburg, Germany) using standard laboratory protocols and $\mathrm{DAB}$ precipitation as end-product. Cell nuclei of immunostained specimens were counterstained with hematoxylin. A consecutive section was stained with HE.

\subsection{Microscopy and general principle of spectral imaging}

A Leica DM RBE light microscope (Leica, Wetzlar, Germany) was equipped for transmission light microscopy including a $63 \times$ Plan-Apo objective (numerical aperture 1.32), and a $100 \mathrm{~W}$ halogen light source (Xenophot HLX; Osram, Berlin, Germany). Spectral images were acquired with Fourier-transform spectroscopy combined with CCD-imaging using the SpectraCube SD200 system (Applied Spectral Imaging, Migdal Ha'Emek, Israel). The general principle of spectral imaging for bright-field microscopy has been described before $[8,10,16,17]$. In spectral imaging, each pixel of the CCD camera is actually one of several hundred thousand of microspectrometers which act simultaneously and independently. Light from the object is collected and collimated by the microscope, enters a Sagnac common-path interferometer [3], is split into two beams which interfere with one and other after a small and variable optical path difference (OPD) has been introduced, and is then focused on the CCD detector. The interference characteristics at each pixel are generated frame by frame by optically changing the OPD, and by recording in memory the digitized signals of each pixel of each frame. All the frames together make up the "cube" of interferogram data, which is subsequently transformed by Fourier-transform calculation into a spectral image "cube", containing the spectrum of each pixel. The computer then processes the spectra with application-specific algorithms, and stores and displays the results.

\subsection{Spectral imaging of bright-field microscopic objects}

Sample illumination was optimized for spectral imaging by operating the light source at $12 \mathrm{~V}$ for daylight color temperature and adjusting the intensity with neutral density filters (Melles Griot, Irvine, CA). Specimens were illuminated predominantly by wavelengths in the human visible range $(450-700 \mathrm{~nm})$ by placing a WG360 UV cut-off filter and a BG38 infrared cutoff filter in the illumination pathway. Spectral images were acquired using Spectral Imaging acquisition software (version 1.2; Applied Spectral Imaging) at default factory settings for bright-field specimens. Absorption spectra were retrieved by spectral pathology software (SpyView 1.0; Applied Spectral Imaging), which can calculate and display the optical density (OD) of the specimen at each wavelength within the chosen range for every pixel in the spectral image. A reference classification library was built by acquiring spectral images of microscopic specimens containing only a single dye, and entering each "pure-dye" spectrum into the library. The single-color reference specimens were processed and stained simultaneously with the multi-color test specimens and were of the same biological material. For analysis of test specimens, SpyView decomposed the acquired spectral images of multi-stain samples into their basic absorbing components [3]. These components were further quantitatively represented in a set of gray-level images in which intensity is proportional to the concentration of the dye. Merging multiple grayscale images in RGB pseudo-colors thus creates a classification image in which the relative concentrations of different pure dyes are readily discernible. At least 10 randomly sampled microscopic fields per specimen were acquired and color classified with SpyView.

\section{Results}

\subsection{Single-color ISH with and without nuclear counterstaining}

The absorption spectra of PO-substrates DAB, AEC, TMB, and AP-substrates FR and NF were measured in 
single-color ISH experiments on T24 cells without nuclear counterstaining (Fig. 1A). The spectra of PO substrates were generally broader than of AP substrates. DAB and AEC spectra were quite similar and so were FR and NF spectra, but they all differed from TMB. In specimens without nuclear staining, spectral classification was accurate for all spots visible by eye (data not shown).

When hematoxylin nuclear stain was used (Fig. 1B), it was present throughout the nucleus, also overlaying the ISH signals. Therefore, the absorption spectrum measured at the ISH signals was mixed with the hematoxylin spectrum (data not shown). Nevertheless, spectral analysis correctly discriminated the DAB-, FRand NF ISH signals from hematoxylin nuclear staining (Figs 2A-L). The colors of small ISH signals, minor binding sites, and large background spots that were not recognizable by eye in heterogeneously stained nuclei, were correctly identified (Fig. 2B). The classification of weak ISH signals was prone to error because of low OD values, or - in case a nuclear staining has been used - low "signal-to-counterstain" ra- tios. We estimate that the ISH signal OD should exceed the counterstain OD at least with a factor 2 to be classified correctly by spectral decomposition. Reversibly, overstained ISH signals compromise recognition of the underlying counterstain (Fig. 2C). When the ISH signals were not overstained, hematoxylin was recognized throughout the nucleus (Figs $2 \mathrm{G}$ and K).

\subsection{Double- and triple-color ISH without nuclear counterstaining}

In double ISH experiments on T24 cells without nuclear counterstaining using the combinations $\mathrm{DAB} / \mathrm{TMB}$ and $\mathrm{DAB} / \mathrm{NF}$ as reporter dyes, ISH spots could be well distinguished. Only the combination $\mathrm{DAB} / \mathrm{AEC}$ did not result in a correct classification of the ISH spots because the dyes have too much spectral overlap (Fig. 1A). Ultimately, spectral analysis of a triple ISH on T24 cells using DAB, TMB, and NF as reporter dyes for the centromeres of chromosome 1 , 7 , and 15 , respectively, resulted in good spectral sepa-
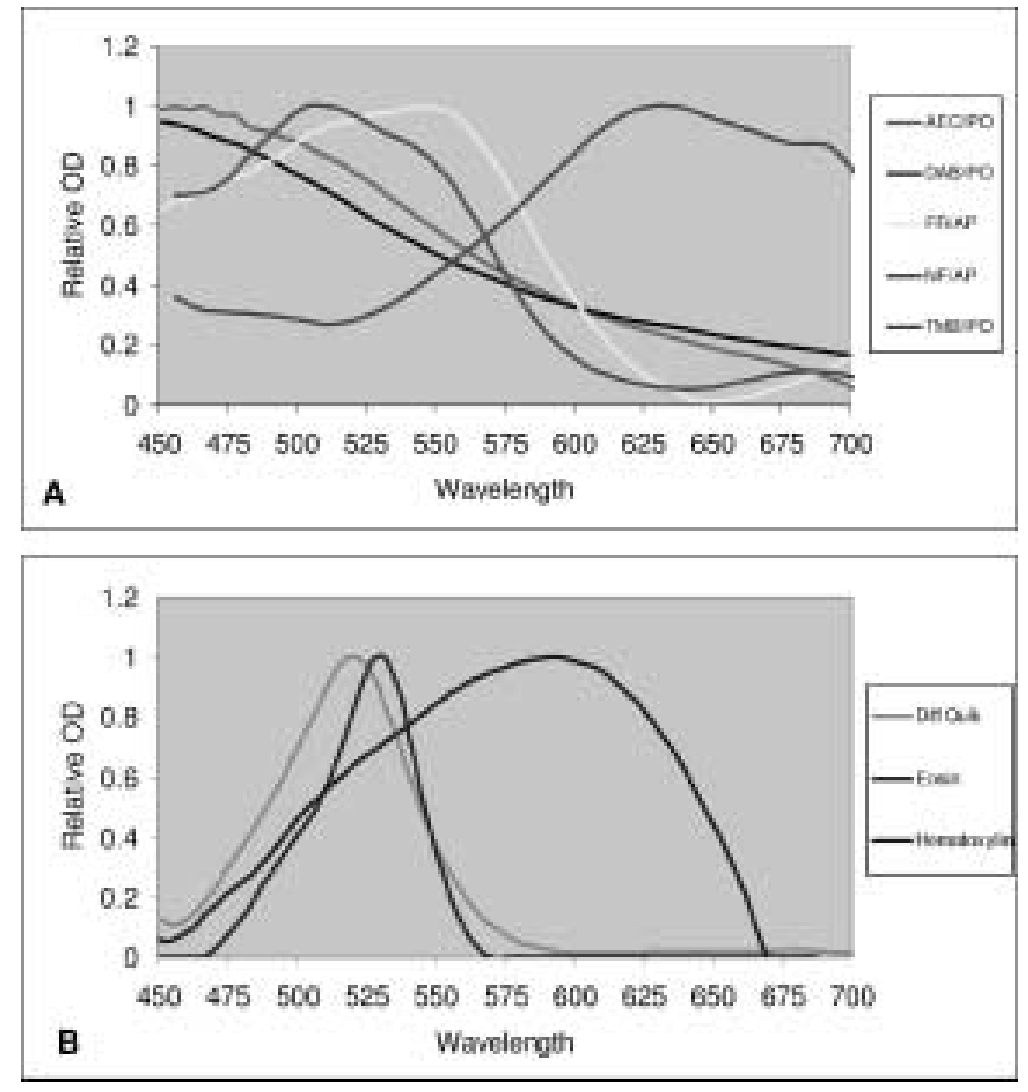

Fig. 1. Chart of pure dye absorption spectra used in ISH and ICC experiments expressed in relative optical density (OD) for all wavelengths between 450 and $700 \mathrm{~nm}$ of (A) enzyme precipitates, and (B) counterstains. 


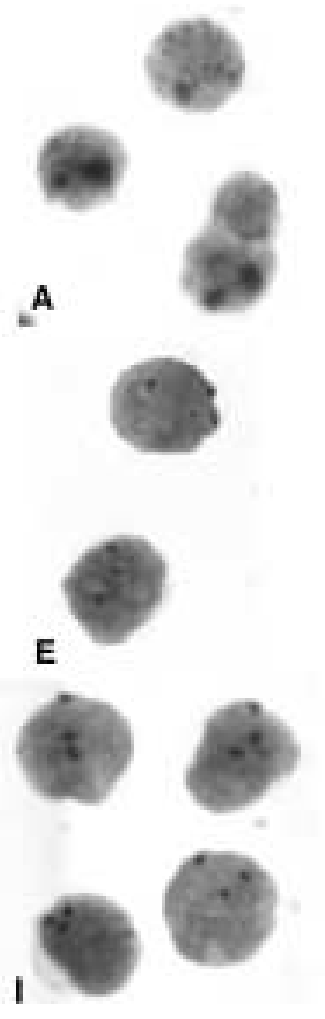

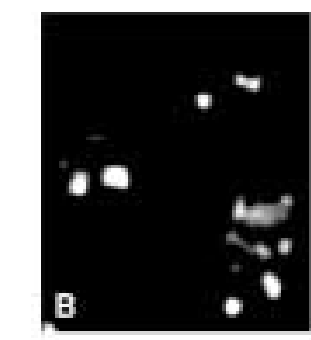
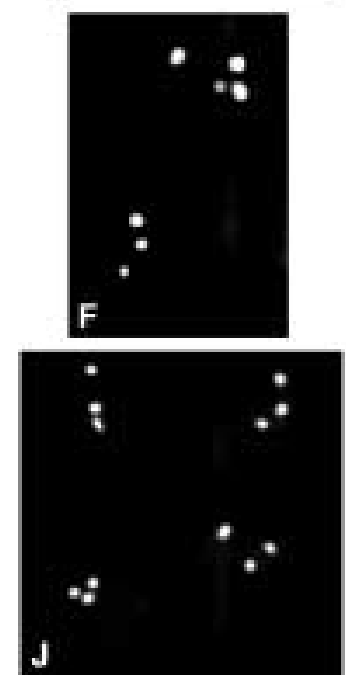
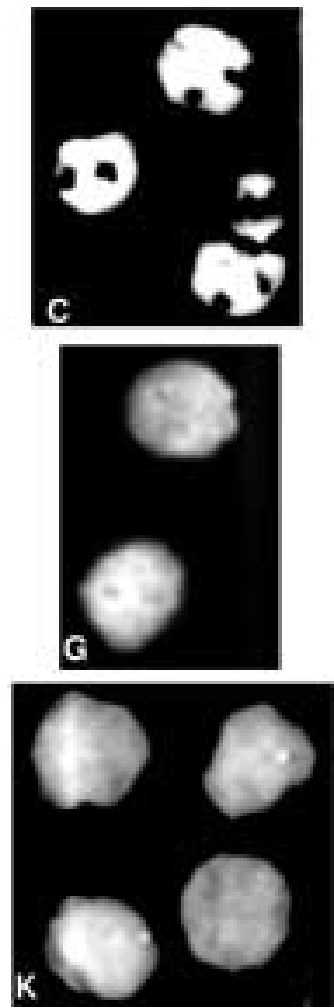
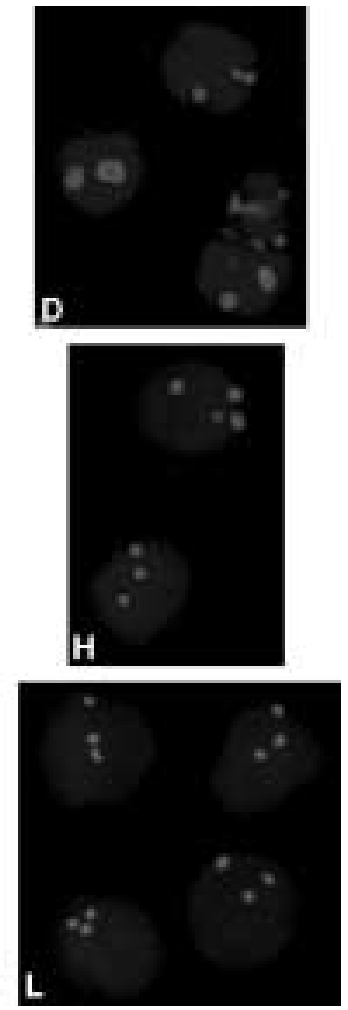

Fig. 2. Spectral analysis of a single-color ISH experiments on T24 cells using a specific probe for the centromere of chromosome 1 visualized with (A-D) DAB, (E-H) New Fuchsin, (I-L) Fast Red, and counterstained with hematoxylin. Panels A, E, and I show the raw microscopic image before spectral analysis. Panels B, F, and J show detection of the ISH signals after spectral decomposition in gray-scale images in which the intensity is proportional to the concentration of the dye. Panels C, G and K show the detection of hematoxylin counterstained nuclei. Panels D, $\mathrm{H}$ and $\mathrm{L}$ are merged gray-scale images in pseudo-colors showing ISH signals in red and nuclei in blue.
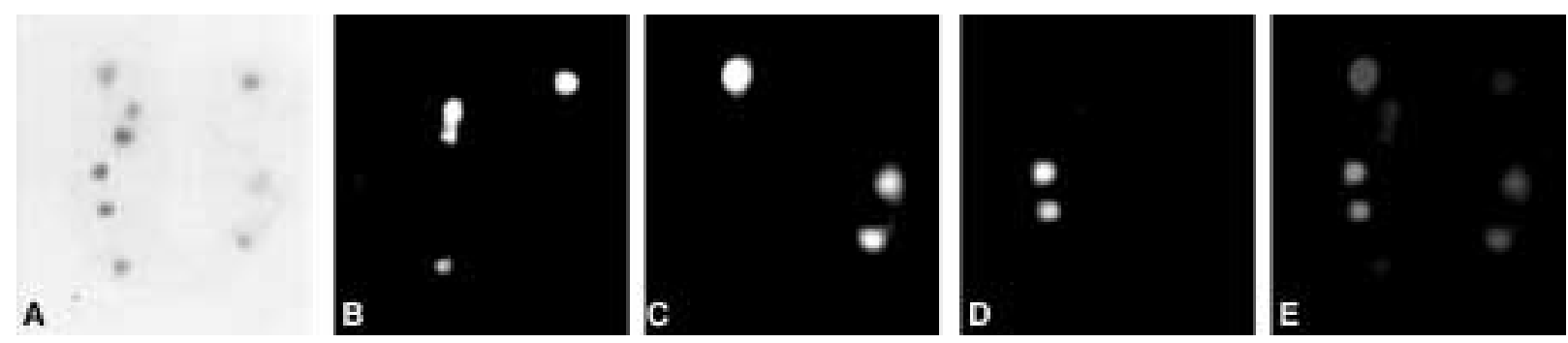

Fig. 3. Spectral analysis of one nucleus of a T24 cell without counterstaining from a triple-color ISH experiment using specific probes for centromeres of chromosome 1, 7, and 15 visualized with DAB, NF and TMB, respectively. The three different colors of the nine ISH signals are difficult to discern in (A) the raw microscopic image before spectral analysis. After spectral decomposition each dye is detected and represented in a gray-scale image (B) DAB, (C) NF and (D) TMB. Merging the three detection images in RGB pseudo-colors results in (E) a color classification image showing DAB in blue, NF in red and TMB in green.

ration and correct classification of the individual dyes (Figs 3A-E).

\subsection{Double-color ISH with counterstaining}

In a double labeling experiment on sperm cells using $\mathrm{X}$ - and Y-chromosome-specific probes visualized with $\mathrm{DAB}$ and $\mathrm{TMB}$, respectively, and cytologically stained with Diff-Quik, clear spectral signatures of all three dyes could be defined (Fig. 4A). Diff-Quik is an eosin derivative (Fig. 1B) that mainly stains the contours of the sperm head and tail. Overlapping sperm cells and minor nuclear staining caused a peak in the spectra of the ISH signals attributable to Diff-Quik 


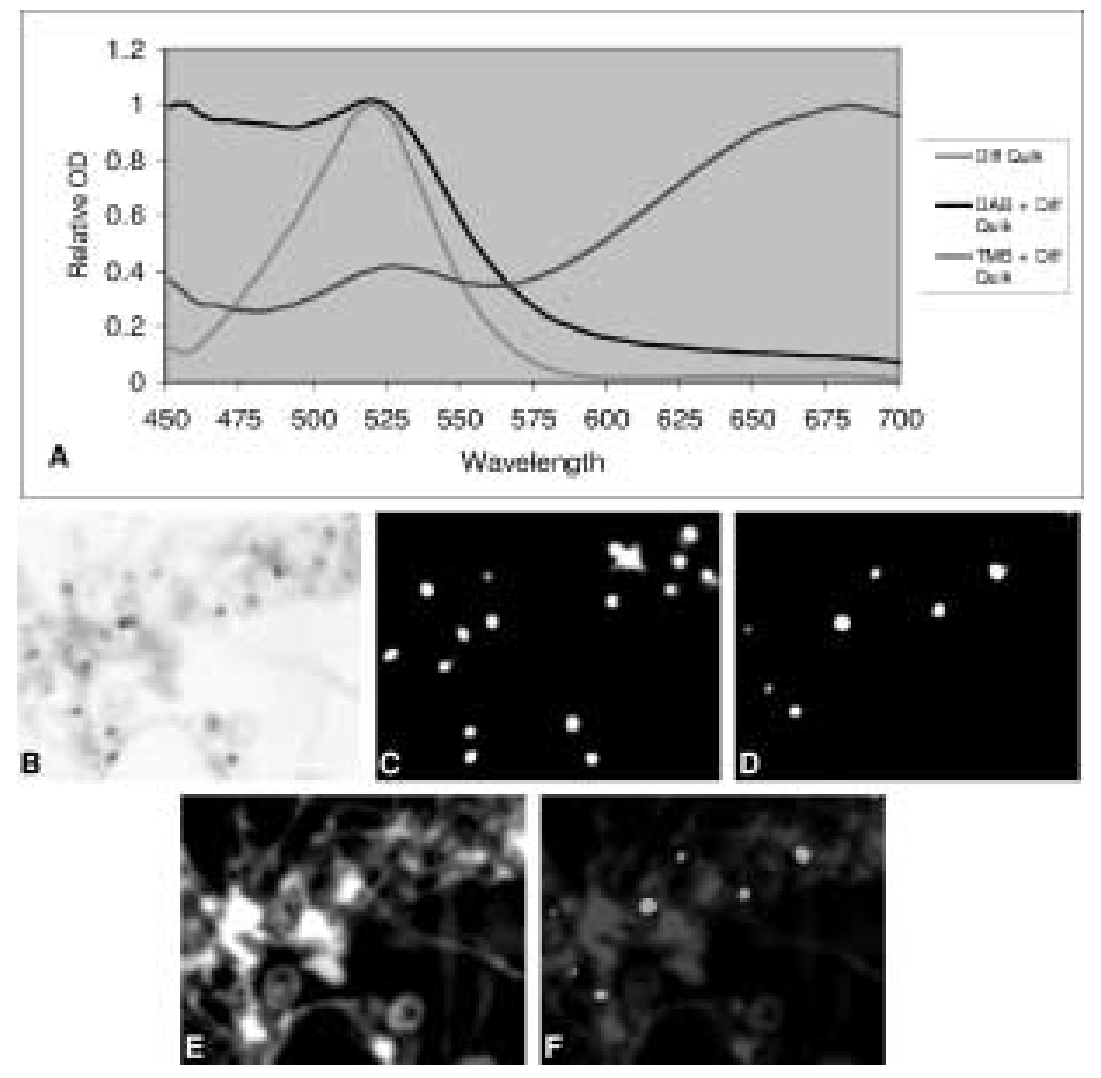

Fig. 4. (A) Chart of DAB and TMB, and Diff-Quik absorption spectra used in an ISH experiment for chromosome $\mathrm{X}$ and $\mathrm{Y}$ specific loci on spermatozoids expressed in relative optical density (OD) for all wavelengths between 450 and $700 \mathrm{~nm}$. Note that the absorption spectra of DAB and TMB measured in the ISH signals contains a peak attributable to Diff-Quik resulting from minor nuclear staining. (B) Raw microscopic image shows a group of overlapping sperm cells. After spectral decomposition the three components are detected and represented in gray-scale images (C) DAB, (D) TMB, and (E) Diff-Quik. Merging the three images in RGB pseudo-colors results in (F) a color classification image showing DAB in blue, TMB in green, and Diff-Quik in red.

(Fig. 4A). Spectrum-based classification using the measured spectra of this specimen -i.e., not of the pure dyes - showed either one $\mathrm{X}$ - or one Y-chromosome ISH spot in each sperm nucleus (Figs 4B-F).

\subsection{Immunostaining for nuclear antigens $\mathrm{Ki}-67$ and TP53}

AgarCyto cell block sections of cervical scraping material were stained with $\mathrm{HE}$, and immunostained for Ki-67 or TP53 using DAB and hematoxylin nuclear staining. Spectral analysis of HE staining showed the hematoxylin staining confined to the nuclei whereas the eosin staining was predominantly found in the cytoplasm, but also co-localizing with hematoxylin in the nuclei (Figs 5B-E). DAB immunostaining and hematoxylin reference spectra were defined in singlestained AgarCyto specimens. Immunopositive nuclei were clearly distinguished from the negative nuclei (Figs 5F-M).

\subsection{Reproducibility}

SpyView utilizes a linear decomposition algorithm for spectral classification. The spectral reference library was built from single-color reference specimens, which were of the same biological material, and processed and stained simultaneously with the multi-color test specimens. This allowed correct classification of every microscopic field (more than 10) from the reference specimens and the multi-color test specimen containing those dyes. Reproducibility of spectral classification using reference spectra from specimens processed and stained at a different time or from different biological material has not been determined. 

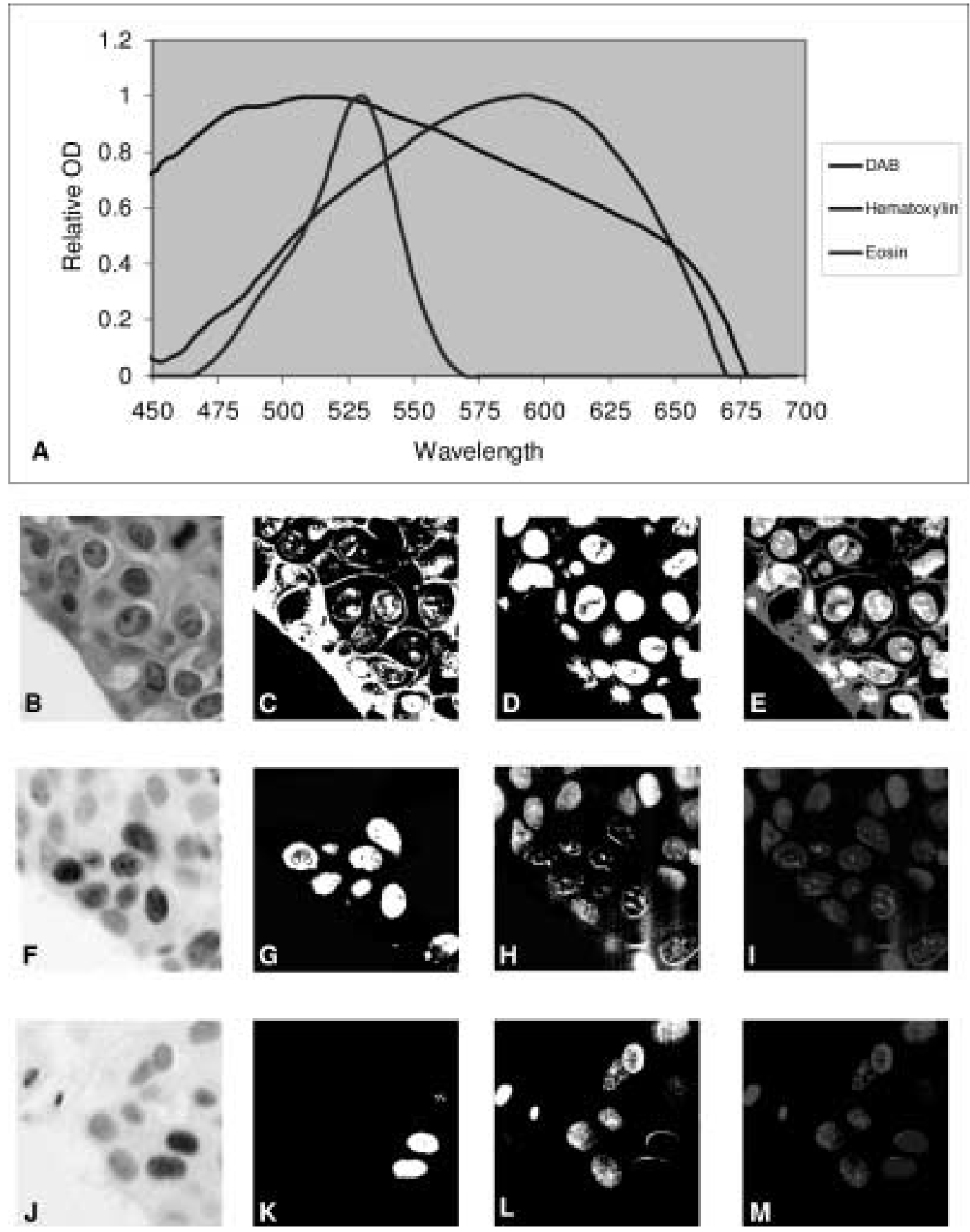

Fig. 5. (A) Chart of DAB, hematoxylin, and eosin pure dye absorption spectra used in ICC experiment on AgarCyto of a cervical scraping from a woman diagnosed with severe dysplasia, expressed in relative optical density (OD) for all wavelengths between 450 and $700 \mathrm{~nm}$. Spectral classification of HE stained paraffin section (B-E) showing the raw microscopic image (B), separate eosin- (C) and hematoxylin- (D) detection in gray-scale images, and merged into an RGB false color image (E). Eosin is shown in purple and found predominantly in the cytoplasm but also in the nucleus. Hematoxylin is shown in green and only found in the nucleus. Pixels in which both eosin and hematoxylin have been detected are shown in white. Spectral analysis of immunodetection for Ki-67 (F-I) and TP53 (J-M). The raw microscope images (F, J) show the brown DAB precipitate for immunopositive nuclei and the blue hematoxylin for immunonegative nuclei. Pixel-by-pixel spectral decomposition resulted in the DAB detection images $(\mathrm{G}, \mathrm{K})$, hematoxylin detection images $(\mathrm{H}, \mathrm{L})$, and the merged images in RGB pseudo-colors, showing DAB immunopositive nuclei in blue and immunonegative nuclei in red (I, M). 


\section{Discussion}

Cytological and histological specimens are routinely stained with permanent dyes that often contain hematoxylin and eosin. The morphology as revealed by these dyes under a transmission light microscope has been the fundament for a pathologist's diagnosis for decades. In particular for the diagnosis of cancer, morphologic screening has been the golden standard for many tissue types and worldwide-standardized diagnostic criteria have been established and implemented into clinical practice.

With increasing knowledge of molecular mechanisms involved in (the cause of) cancer, new possibilities are emerging to refine pathologic diagnosis. Complementing the morphologic data with molecular data in situ can refine the diagnosis and staging of certain types of cancer. These include (a) phenotypic protein expression data obtained by means of immunocytochemistry (ICC) with antibodies against products of commonly deregulated oncogenes or tumor suppressor genes, (b) protein function assays by enzyme histochemical procedures, and (c) molecular cytogenetic detection by in situ hybridization (ISH) of genomic alterations of chromosomes or chromosome loci. For the simultaneous assessment of morphologic and molecular data in situ, specimens must be prepared using multiple stains that can be distinguished based on their color under a light microscope. Advances in cytochemical detection methods and microscopic imaging have enabled the application of ICC and ISH for multiple targets on the same specimen. The obvious method of choice for multi-color analysis could be fluorescence microscopy, but for routine pathological diagnostic purposes fluorescence microscopy is not preferred because (a) fluorescent dyes bleach fast, hampering long-term storage, revision, and retrospective studies, (b) crucial morphological information might be missed because combinations with routine stains are often not possible, and (c) some tissue types show high auto-fluorescence obscuring the cytochemical detection signals. To circumvent these problems, multicolor bright-field microscopy using enzyme precipitates of different color has been developed in the past few years for simultaneous detection of multiple genes and gene-products $[21,22]$.

Digital microscopy, using three-CCD color video cameras, and advanced software packages for color recognition based on red, green, blue (RGB)-algorithms and derivatives thereof such as hue, saturation, intensity (HSI)- , and hue, saturation, density (HSD)- algorithms are able to identify up to three non colocalizing colors correctly. The HSD method provides the best classifiers for proper segmentation and object recognition [24]. Despite the advances in color image analysis, the spectral resolution of three-CCD color video images remains limited.

In the present study, we explored the possibilities of spectral imaging for pixel-by-pixel color classification of multi-color ISH and ICC specimens. Our present data show that spectral imaging is able to accurately discriminate dyes with partial overlapping spectra, enabling, thus far, the color-classification of pathological specimens containing three different chromogenic dyes.

Pixel-by-pixel color classification and pseudocolorization greatly facilitates interpretation of the multi-color specimen compared to microscopic examination with the human eye. Color discrimination by the unaided eye of small ISH signals in a counterstained cell or nucleus, as performed on the sperm cells, often faces problems. The dyes included in our study are the most commonly used in immunostaining and ISH for bright-field microscopy. Other chromogenic dyes are available that may even have more favorable spectral characteristics to increase the number of detection targets. Future experiments should show the possibilities and limits of spectral imaging concerning (a) colocalization of immunostains and/or ISH spots (spectral resolution), (b) classify spots with lower density (sensitivity) or smaller size (topographical resolution), and (c) discrimination of more than three targets simultaneously (multiplicity). A study in which these issues are being addressed and compared to image analysis of three-color CCD video images is underway.

In previous reports on bright-field spectral imaging $[8,11,16]$, specimens were analyzed by basic spectral analysis software SpCube. This software uses pixel classification by a minimal square error algorithm, which in our experience does not allow the spectral analysis of multiple microscopic views from the same specimen using the same spectral reference library. The new SpyView software uses a "pure dye" spectrum library acquired from single-color reference specimens, for pixel classification by a linear decomposition algorithm [3]. This enables the analysis of a series of images of different test specimens, on the condition that the reference and test specimens were processed and stained simultaneously and contain the same biological material. SpyView also features "spectral unmixing" for true-color representation of a classified image, as well as pseudo-colorization and separate vi- 
sualization of each color component of the image in a gray-scale or true-color image (not shown).

The differences in spectrum of TMB in T24 cells and in sperm cells (Figs 1A and 4A, respectively) underline the fact that variations in spectral characteristics may occur in specimens which were processed and stained under different circumstances. In this case, color differences were due to different Tungsten stabilization conditions. It cannot be ruled out that spectral differences could also be introduced by different types of tissue, different conditions for ISH, immunocytochemistry, or enzyme reactions, or possible other unknown factors.

For widespread use of spectral imaging in quantitative pathology, the pixel-by-pixel spectral information should be adapted to a segmentation algorithm for stained object identification, such as tissue compartments, cell populations and subcellular components. Correct segmentation could be a starting point for comprehensive quantitative image analysis of multi-color stained specimens used in pathologic diagnosis. In this study, the detection of differentially stained nuclei and ISH signals was performed with high accuracy and might allow enumeration of multi-color interphase ISH signals. Also, our data show that accurate and comprehensive quantitative measurements of differentially immunostained cellular features might become feasible.

In summary, bright-field spectral imaging analyzes absorption spectra with a high precision and maintains spatial information. The use of cytological stains doesn't hamper spectral analysis and will greatly facilitate microscopic evaluation. Spectral imaging offers a higher color resolution than RGB-color video CCD imaging, providing the requirements for reliable and user-friendly color discrimination of multi-color specimens. Spectral imaging of pathological specimens, or spectral pathology (SPY), has great potential for multiparameter analysis of pathological specimens and may provide a solution for automated object segmentation in digital microscopy.

\section{Acknowledgements}

The authors wish to thank Dr. J. Kaufman (ASI, Israel) for spectral imaging support, Dr. E. Martini (University Maastricht, The Netherlands) for multi-color staining of sperm specimens, and S.Wienk (UMC Nijmegen, The Netherlands) for preparing and staining of cervical AgarCyto specimens. This work was supported in part by grant 97-1486 from the Dutch Cancer Society.

\section{References}

[1] J. Bulten, P.J. Poddighe, J.C. Robben, J.H. Gemmink, P.C. de Wilde and A.G. Hanselaar, Interphase cytogenetic analysis of cervical intraepithelial neoplasia, Am. J. Pathol. 152 (1998), 495-503.

[2] J. Bulten, J.A. Van der Laak, J.H. Gemmink, M.M. Pahlplatz, P.C. de Wilde and A.G. Hanselaar, MIB1, a promising marker for the classification of cervical intraepithelial neoplasia, J. Pathol. 178 (1996), 268-273.

[3] Y. Garini, N. Katzir, D. Cabib, R.A. Buckwald, D. Soenksen and Z. Malik, Spectral bio-imaging, in: Fluorescence Imaging Spectroscopy and Microscopy, Chemical Analysis Series, Vol. 137, John Wiley \& Sons, New York, 1996, pp. 84-124.

[4] A.H. Hopman, S. Claessen and E.J. Speel, Multi-colour brightfield in situ hybridisation on tissue sections, Histochem. Cell Biol. 108 (1997), 291-298.

[5] A.H. Hopman, F.C. Ramaekers, A.K. Raap, J.L. Beck, P. Devilee, M. Van der Ploeg and G.P. Vooijs, In situ hybridization as a tool to study numerical chromosome aberrations in solid bladder tumors, Histochemistry 89 (1988), 307-316.

[6] H.M. Kerstens, J.C. Robben, P.J. Poddighe, W.J. Melchers, H. Boonstra, P.C. de Wilde, M.V. Macville and A.G. Hanselaar, AgarCyto: a novel cell-processing method for multiple molecular diagnostic analyses of the uterine cervix, J. Histochem. Cytochem. 48 (2000), 709-718.

[7] T. Knutsen and T. Ried, SKY: A comprehensive diagnostic and research tool. A review of the first 300 published cases, J. Assoc. Genetic Technologists 26 (2000), 3-15.

[8] R.M. Levenson and D.L. Farkas, Digital spectral imaging for histopathology and cytopathology, Proc. SPIE 2983 (1997), 123-135.

[9] C. MacAulay, H. Tezcan and B. Palcic, Adaptive color basis transformation. An aid in image segmentation, Analyt. Quant. Cytol. Histol. 11 (1989), 53-58.

[10] Z. Malik, D. Cabib, R.A. Buckwald, A. Talmi, Y. Garini and S.G. Lipson, Fourier transform multipixel spectroscopy for quantitative cytology, J. Microscopy 182 (1996), 133-140.

[11] Z. Malik, C. Rothmann, T. Cycowitz, Z.J. Cycowitz and A.M. Cohen, Spectral morphometric characterization of B-CLL cells versus normal small lymphocytes, J. Histochem. Cytochem. 46 (1998), 1113-1118.

[12] E. Martini, J.P. Geraedts, I. Liebaers, J.A. Land, G.L. Capitanio, F.C. Ramaekers and A.H. Hopman, Constitution of semen samples from XYY and XXY males as analysed by in-situ hybridization, Humun Reproduction 11 (1996), 1638-1643.

[13] E. Martini, E.J. Speel, J.P. Geraedts, F.C. Ramaekers and A.H. Hopman, Application of different in-situ hybridization detection methods for human sperm analysis, Human Reproduction 10 (1995), 855-861.

[14] T. Ried, Interphase cytogenetics and its role in molecular diagnostics of solid tumors, Am. J. Pathol. 152 (1998), 325-327.

[15] P. Rostagno, I. Birtwisle, F. Ettore, A. Courdi, J. Gioanni, M. Namer and C. Caldani, Immunohistochemical determination of nuclear antigens by colour image analysis: application for labelling index, estrogen and progesterone receptor status in breast cancer, Analyt. Cell. Pathol. 7 (1994), 275-287. 
[16] C. Rothmann, I. Bar-Am and Z. Malik, Spectral imaging for quantitative histology and cytogenetics, Histol. Histopathol. 13 (1998), 921-926.

[17] C. Rothmann, A.M. Cohen and Z. Malik, Chromatin condensation in erythropoiesis resolved by multipixel spectral imaging: differentiation versus apoptosis, J. Histochem. Cytochem. 45 (1997), 1097-1108.

[18] E. Schröck, S. du Manoir, T. Veldman, B. Schoell, J. Wienberg, M.A. Ferguson-Smith, Y. Ning, D.H. Ledbetter, I. Bar-Am, D. Soenksen, Y. Garini and T. Ried, Multicolor spectral karyotyping of human chromosomes, Science 273 (1996), 494-497.

[19] E. Schröck and H. Padilla-Nash, Spectral karyotyping and multicolor fluorescence in situ hybridization reveal new tumorspecific chromosomal aberrations, Semin. Hematol. 37 (2000), 334-347.

[20] E. Schröck, T. Veldman, H. Padilla-Nash, Y. Ning, J. Spurbeck, S. Jalal, L.G. Shaffer, P. Papenhausen, C. Kozma, M.C. Phelan, E. Kjeldsen, S.A. Schonberg, P. O’Brien, L. Biesecker, S. du Manoir and T. Ried, Spectral karyotyping refines cytogenetic diagnostics of constitutional chromosomal abnormalities, $\mathrm{Hu}$ man Genetics 101 (1997), 255-262.

[21] E.J. Speel, Robert Feulgen Prize Lecture 1999. Detection and amplification systems for sensitive, multiple-target DNA and RNA in situ hybridization: looking inside cells with a spectrum of colors, Histochem. Cell Biol. 112 (1999), 89-113.
[22] E.J. Speel, M.P. Jansen, F.C. Ramaekers and A.H. Hopman, A novel triple-color detection procedure for brightfield microscopy, combining in situ hybridization with immunocytochemistry, J. Histochem. Cytochem. 42 (1994), 1299-1307.

[23] E.J. Speel, B. Schutte, J. Wiegant, F.C. Ramaekers and A.H. Hopman, A novel fluorescence detection method for in situ hybridization, based on the alkaline phosphatase-fast red reaction, J. Histochem. Cytochem. 40 (1992), 1299-1308.

[24] J.A. Van der Laak, M.M. Pahlplatz, A.G. Hanselaar and P.C. de Wilde, Hue-saturation-density (HSD) model for stain recognition in digital images from transmitted light microscopy, $C y$ tometry 39 (2000), 275-284.

[25] T. Veldman, C. Vignon, E. Schröck, J.D. Rowley and T. Ried, Hidden chromosome abnormalities in haematological malignacies detected by multicolour spectral karyotyping, Nature Genetics 15 (1997), 406-410.

[26] F. Willemse, M. Nap, L.B. de Kok and H.F. Eggink, Image analysis in immunohistochemistry. Factors with a possible influence on the performance of VIDAS version 2.0, a commercially available true color image analysis system, Analyt. Quant. Cytol. Histol. 15 (1993), 136-143. 


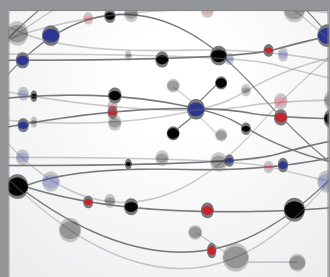

The Scientific World Journal
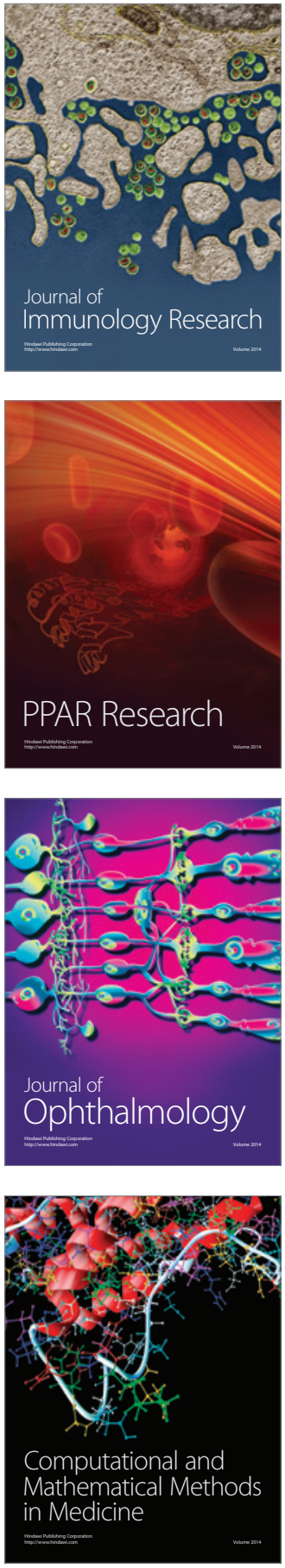

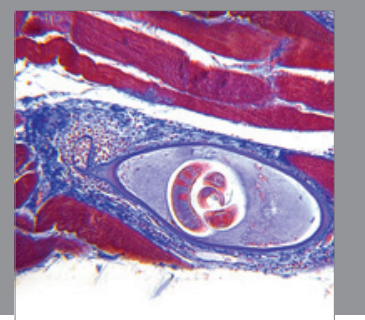

Gastroenterology

Research and Practice
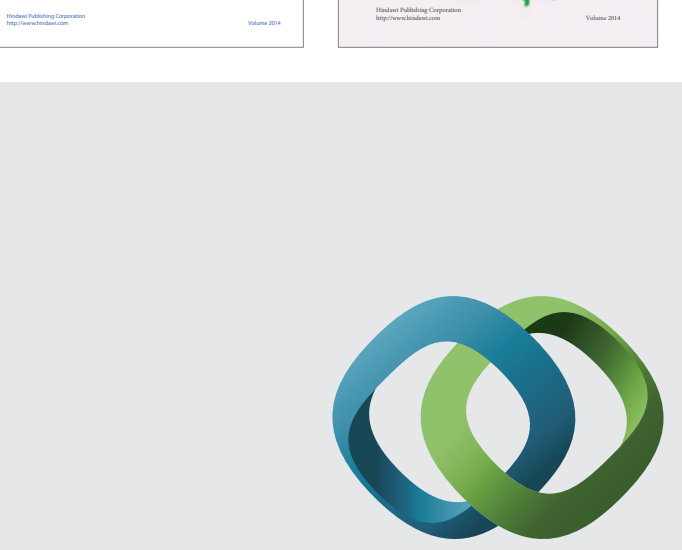

\section{Hindawi}

Submit your manuscripts at

http://www.hindawi.com
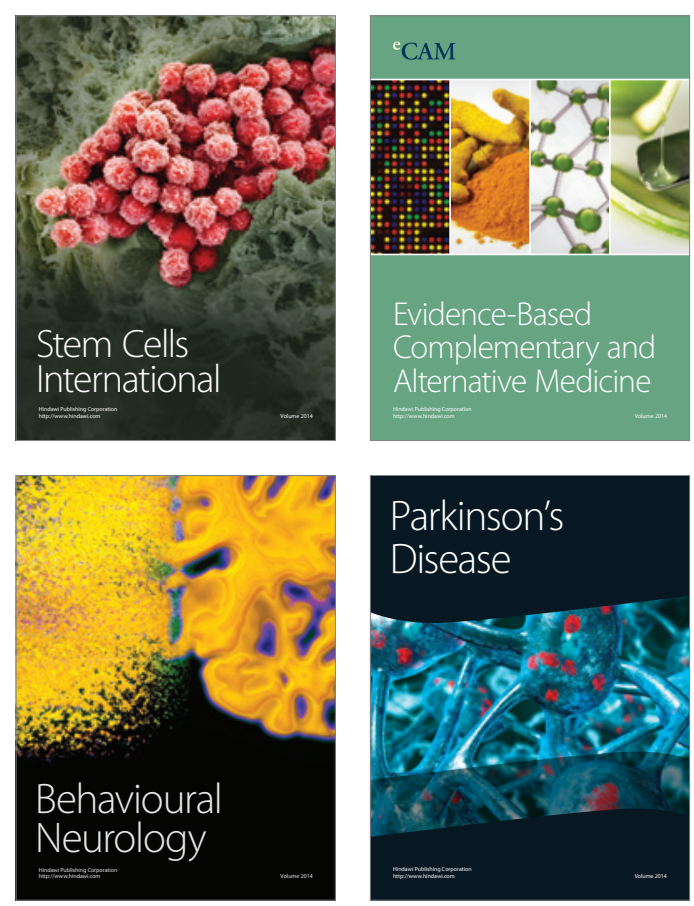

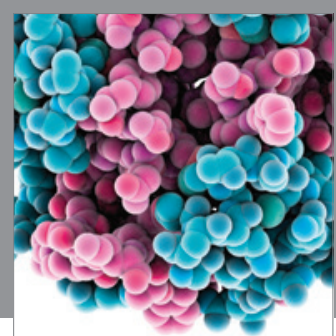

Journal of
Diabetes Research

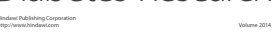

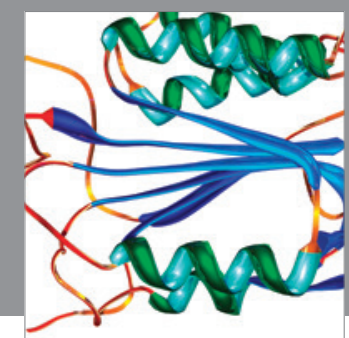

Disease Markers
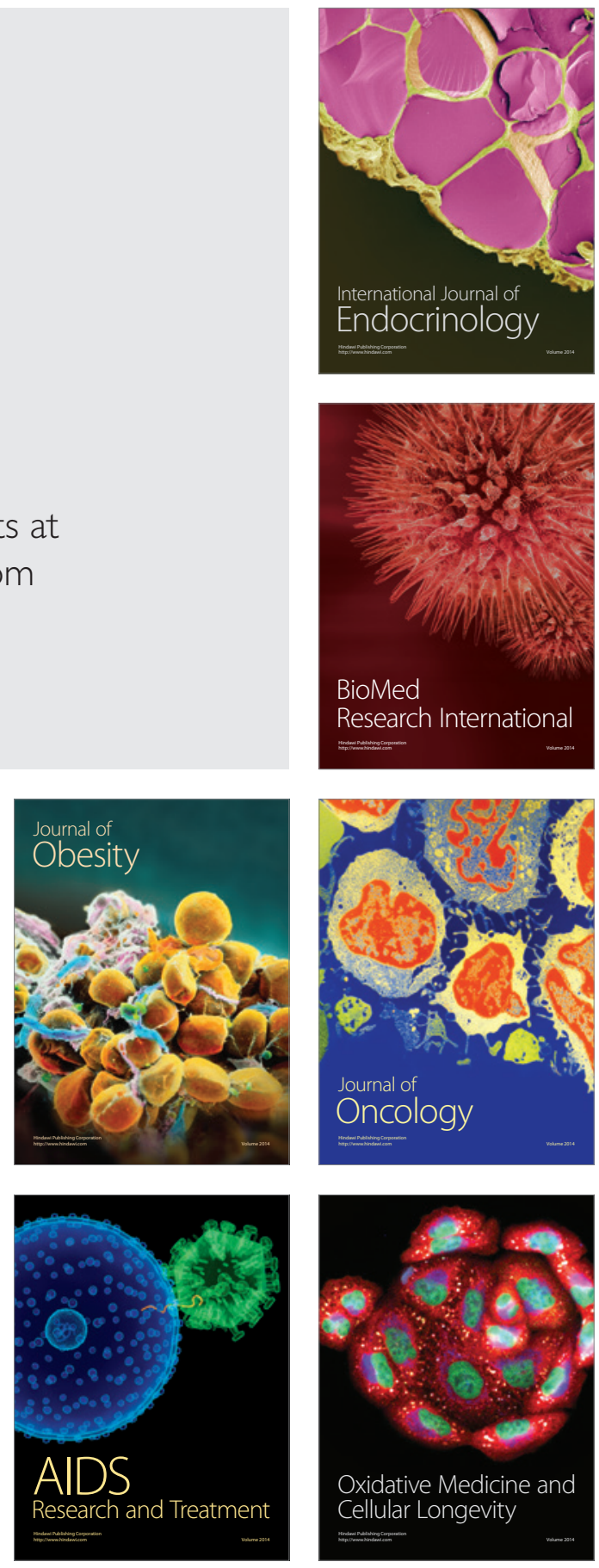